\title{
Emploi du 'futur' dans le français parlé des élèves d'immersion française
}

TERRY NADASDI

Université de l'Alberta

\author{
RAYMOND MOUGEON \\ Université York
}

KATHERINE REHNER

Université de Toronto

(Received February 2002; revised June 2002)

\section{A B S T RAC T}

Notre étude porte sur l'acquisition des variantes du futur par les élèves d'immersion. Tous comme les locuteurs LI, ces élèves emploient trois variantes (le futur périphrastique; le futur fléchi et le présent de l'indicatif) et ils le font au même niveau de fréquence: le futur périphrastique est de loin la plus fréquente. Nous discutons des facteurs éducatifs et sociologiques auxquels on peut attribuer ces résultats inattendus. Ceci dit, les élèves d'immersion expriment aussi parfois le futur avec des formes verbales non natives qui font l'objet d'une étude spéciale. Nous terminons par une micro-étude des variantes du futur périphrastique à la I ère personne du singulier.

\section{INTRODUCTION}

La présente étude s'inscrit dans le cadre d'un projet de recherche centré sur l'apprentissage de la variation sociostylistique du français parlé par les élèves d'immersion française en Ontario. Notre étude porte sur les différentes constructions verbales qu'emploient ces élèves pour exprimer la notion grammaticale de futur. Dans la première partie de notre article, nous passons en revue les variantes du futur que l'on trouve en français canadien LI $_{\text {I }}$ et nous vérifions si les élèves d'immersion utilisent ces variantes. Nous vérifions aussi si ces élèves respectent les contraintes linguistiques et sociales qui sont associées à ces variantes en français LI. La deuxième partie est consacrée aux «variantes» non natives employées par ces élèves pour exprimer la notion de futur. La troisième partie est centrée sur les formes du futur périphrastique employées à la I ${ }^{\text {ère }}$ personne du singulier (par ex.: je vais, je vas, m'as). Nous vérifions, entre autres, si les élèves d'immersion emploient deux variantes informelles typiques du parler des locuteurs Li du français canadien. 
2 bRef RÉsumé Des objectifs de notre projet

DE RECHERCHE

Notre recherche sur l'acquisition de la variation sociostylistique par les élèves d'immersion française fait suite à une série d'études linguistiques sur le rendement en français de ces élèves. Ces études, dont le lecteur peut trouver un exemple dans Harley (1992), ont constaté que si les élèves d'immersion ont une bonne maitrise du français parlé, elle est 'moins que native'. On observe notamment dans leur discours oral un nombre non négligeable d'erreurs récurrentes. Compte tenu de ces résultats, lorsque nous avons démarré notre recherche en I996, nous ne nous attendions pas à trouver que les élèves d'immersion possèdent un répertoire de variantes sociostylistiques comparable à celui des locuteurs natifs du français, ni à ce qu'ils soient toujours capables d'utiliser ces variantes en respectant les contraintes linguistiques ou extra-linguistiques qui pèsent sur leur emploi en français LI.

Trois facteurs principaux nous ont poussé à entreprendre cette recherche:

- Dans ses directives pour l'enseignement du français dans les programmes d'immersion, le ministère de l'Éducation de l'Ontario souligne l'importance de la maitrise des niveaux de langue et de la connaissance des variétés du français local. Notre recherche était donc susceptible de fournir des données utiles pour l'élaboration de matériel pédagogique centré sur ces deux objectifs.

- Par le biais de notre recherche nous voulions contribuer au développement d'un nouveau champ d'études centré sur l'acquisition de la variation par les apprenants des langues secondes et sur les facteurs internes et externes qui influencent cette acquisition (cf. Bayley et Preston, I996, pour plus d'informations sur les premières études dans le domaine).

- Dans des recherches antérieures nous avons analysé la variation du français parlé d'adolescents franco-ontariens, scolarisés en français, mais élevés dans des foyers où l'on ne maintient guère cette langue (cf. entre autres, Mougeon et Beniak, I99I). Notre recherche sur l'apprentissage de la variation par les élèves d'immersion française nous permettait donc de pousser plus loin la réflexion sur la compétence sociolinguistique des locuteurs du français qui utilisent cette langue dans un nombre restreint de situations de communication.

Notre recherche sur l'acquisition de la variation sociostylistique du français parlé par les élèves d'immersion a pour but de fournir des éléments de réponse aux questions suivantes:

- Est-ce que, sur un point particulier de la structure de la langue, les élèves d'immersion emploient les mêmes variantes que les locuteurs Li du français canadien et est-ce qu'ils emploient ces variantes avec une fréquence comparable à celle que l'on peut observer dans le français parlé de ces mêmes locuteurs?

- Est-ce que l'emploi des variantes par les élèves d'immersion est influencé par: a) les mêmes facteurs internes et externes qui influent sur l'usage de ces variantes 
par les locuteurs LI du français canadien (p. ex. l'appartenance socio-économique et le sexe) et b) d'autres facteurs externes qui sont propres aux élèves d'immersion (p. ex. le niveau de langue employé par leurs enseignants en salle de classe, leurs contacts avec des locuteurs natifs, leur langue première, etc.)?

Les cas de variation sur lesquels porte notre recherche ont déjà fait l'objet d'études sociolinguistiques à partir de corpus de français oral LI, recueillis à l'aide d'entrevues semi-dirigées enregistrées semblables à celles que nous avons effectuées pour notre propre recherche. Ces études nous permettent donc de comparer le français parlé des élèves d'immersion à celui des locuteurs natifs du français avec des données recueillies dans des situations de communication similaires.

Les études sur la variation du français parlé Li que nous utilisons dans le cadre de notre recherche reposent sur des corpus de français québécois (principalement les corpus du français parlé à Montréal: corpus Sankoff et Cedergren et corpus Thibault et Vincent) ou ontarien (le corpus du français parlé dans les quatre localités de Cornwall, Hawkesbury, North Bay et Pembroke: corpus Mougeon et Beniak et celui du français parlé à Ottawa-Hull: corpus de Poplack).

Étant donné que les élèves qui sont visés par notre recherche apprennent surtout le français en contexte scolaire (cf. plus bas), nous portons une attention particulière à l'influence de la langue des enseignants et des manuels de français. Pour ce faire, nous utilisons à des fins comparatives un corpus qui nous fournit des données sur la langue employée en salle de classe par un échantillon de sept enseignants ontariens travaillant dans des programmes d'immersion française du Grand Toronto et de la région d'Ottawa (Allen et al., I987). Nous analysons aussi la langue employée dans deux manuels de français communément utilisés dans les programmes d'immersion des districts scolaires du Grand Toronto. ${ }^{1}$

Pour mieux saisir les résultats de la présente étude, nous allons faire un bref survol des résultats des travaux antérieurs sur l'apprentissage de la variation sociostylistique du français parlé par les élèves d'immersion.

3 RECHERCHES ANTÉRIEURES SUR L'ACQUiSITION DE LA
VARIATION SOCIOSTYLISTIQUE PAR LES ÉLÈVES D’IMMERSION

On peut résumer les résultats des recherches antérieures à l'aide des tableaux I et 2 qui fournissent des informations portant sur les études pertinentes. Le lecteur trouvera des renseignements plus détaillés dans Mougeon, Nadasdi et Rehner (à paraître).

${ }^{1}$ Il s'agit des manuels inclus dans les séries Portes ouvertes sur mon pays et Capsules (cf., Deslauriers et Gagnon, I995; Deslauriers et Gagnon, I997; Le Dorze et Morin, 1994 et Roy Nicolet et Jean-Côté, 1994). La série Portes ouvertes a été élaborée pour l'enseignement $\mathrm{du}$ français en immersion. La série Capsules a été préparée pour les élèves québécois, cependant on l'utilise aussi dans plusieurs programmes d'immersion en Ontario. 
Tableau I. Résultats relatifs à la fréquence des variantes dans le français parlé des élèves d'immersion

Les élèves d'immersion n'emploient pas ou emploient très rarement les variantes qui sont typiques des variétés de français populaires

Les élèves d'immersion emploient les variantes informelles courantes, toutefois, leur emploi de ces variantes est nettement moins fréquent que dans le parler des locuteurs LI

Les élèves d'immersion emploient les variantes formelles nettement plus souvent que les locuteurs $\mathrm{L}_{\mathrm{I}}$
Mougeon et Rehner (200I); Rehner, Mougeon et Nadasdi (200Ia);

Nadasdi et McKinnie (à paraître)

Rehner et Mougeon (I999); Rehner, Mougeon et Nadasdi (à paraitre);

Uritescu, Nadasdi, Mougeon et

Rehner (200I); Mougeon, Nadasdi, Rehner, et Uritescu (2002)

Rehner et Mougeon (1999); Rehner, Mougeon et Nadasdi (200I);

Rehner, Mougeon et Nadasdi (à paraître)

Tableau 2. Effet des facteurs linguistiques et extra-linguistiques sur l'emploi des variantes par les élèves d'immersion

Les contacts avec les locuteurs $\mathrm{LI}_{\mathrm{I}}$

du français ont un impact positif sur l'emploi des variantes informelles courantes ou populaires par les élèves d'immersion

La longueur de l'immersion en français peut avoir un impact positif sur l'emploi de certaines variantes formelles

Les élèves d'immersion du sexe féminin font un emploi moins fréquent que ceux du sexe masculin de certaines variantes populaires et/ou informelles courantes Les élèves d'immersion provenant des couches sociales basses emploient plus souvent certaines variantes populaires et/ou informelles courantes que ceux des couches sociales élevées

Les élèves d'immersion surutilisent certaines variantes qui ont une contrepartie similaire dans leur LI

Le répertoire et l'usage des variantes par les élèves d'immersion reflètent la langue des enseignants et/ou des manuels de français

Les élèves d'immersion ne respectent pas certaines des contraintes linguistiques de la variation observables dans le parler des locuteurs LI

Les élèves d'immersion ont une maitrise imparfaite des contraintes stylistiques de la variation
Lapkin, Hart et Swain (1995); Rehner et Mougeon (I999); Rehner, Mougeon et Nadasdi (à paraître); Mougeon et Rehner (200I)

Swain et Lapkin (I990)

Mougeon et Rehner (200I); Rehner, Mougeon et Nadasdi (à paraitre)

Rehner, Mougeon et Nadasdi (à paraître); Knaus et Nadasdi (200I)

Rehner, Mougeon et Nadasdi (à paraitre); Mougeon et Rehner (200I); Rehner, Mougeon et Nadasdi (200I); et Nadasdi et McKinnie (à paraitre)

Rehner et Mougeon (I999); Rehner, Mougeon et Nadasdi (200I)

Rehner et Mougeon (I999); Nadasdi (200I); Knaus et Nadasdi (200I)

Swain et Lapkin (I990); Lyster, (1994); Uritescu, Nadasdi, Mougeon et Rehner (200I) 


\section{MÉTHODOLOGIE}

La présente étude et le projet de recherche dans lequel elle s'inscrit, reposent sur un échantillon de $4 \mathrm{I}$ élèves inscrits en $9^{\text {ème }}$ et en $\mathrm{I}_{2}{ }^{\text {ème }}$ année (c'est-à-dire âgés de $\mathrm{I} 3$ à I 8 ans) dans un programme d'immersion appelé français intensif ('Extended French'). Dans les programmes de français intensif du district scolaire du Grand Toronto où nous avons recueilli nos données, les élèves suivent la moitié de leurs cours en français de la $5^{\text {ème }}$ année à la $8^{\text {ème }}$ année. À partir de la $9^{\text {ème }}$ année, la proportion des cours enseignés en français n'est plus que de $20 \%$. Aucun des parents de nos 4I élèves d'immersion n'est un locuteur natif du français et ne parle français à la maison. Ceci dit, nos élèves ne proviennent pas pour autant en majorité d'un foyer unilingue anglophone. En fait, comme le montre le tableau 3, 5I\% de nos élèves proviennent d'un foyer où, en plus de l'anglais, on parle une autre langue. Près de $40 \%$ des élèves qui viennent d'un foyer bilingue parlent une langue romane (l'italien ou l'espagnol), le reste de ces élèves parle des langues non romanes indoeuropéennes ou non (p. ex. l'allemand, le serbo-croate, le polonais, le vietnamien, le coréen, le tagalog).

Pour ce qui est de l'emploi actif du français, dans la vie quotidienne, à l'école et en dehors de l'école, nous avons trouvé les tendances suivantes. La seule situation où les élèves font un usage actif non négligeable du français est lorsqu'ils communiquent avec leur enseignant en salle de classe. Lorsqu'ils communiquent avec leurs copains de classe, en classe, ils emploient parfois le français, mais en dehors de la classe, leur emploi de cette langue est marginal. On doit préciser que le programme d'immersion dans lequel sont inscrits nos élèves se trouve dans une école secondaire anglaise où la grande majorité des élèves reçoit un enseignement totalement en anglais et où l'anglais est la langue de communication très largement dominante, entre les élèves et entre le personnel et les élèves.

En ce qui a trait à la communication avec les habitants de leur voisinage ou de leur quartier, nous avons trouvé une tendance similaire. Nos élèves emploient rarement (ou jamais) le français, résultat qui reflète la rareté des francophones dans la localité où ils vivent. ${ }^{2}$

Le tableau 3 montre aussi que les élèves sont répartis à peu près également en $9^{\text {ème }}$ et en $\mathrm{I}_{2}{ }^{\text {ème }}$ année, qu'il y a plus de filles que de garçons, que plus de la moitié des élèves sont issus d'une famille appartenant à la moyenne et petite bourgeoisie et que le reste des élèves provient d'une famille appartenant à la classe ouvrière supérieure. La prépondérance des élèves du sexe féminin et provenant des couches sociales plus élevées est un trait typique de la population estudiantine des programmes d'immersion. La majorité des élèves a été éduquée en français de 28 à $38 \%$ du temps. La plupart des élèves n'écoutent jamais les médias francophones. Toutefois, parmi les élèves qui écoutent ces médias, on trouve une plus grande proportion d'élèves de $12{ }^{\text {ème }}$ année. Les élèves de $12{ }^{\text {ème }}$ année ont aussi séjourné plus longtemps dans un milieu francophone que les élèves de $9^{\text {ème }}$ année. En fait $70 \%$ des élèves de $\mathrm{I}^{\text {ème }}$ année ont séjourné plus d'une semaine dans un milieu

${ }^{2}$ Ces informations ont été receuillies par le biais d'un questionnaire écrit. 
Tableau 3. Caractéristiques principales de l'échantillon d'élèves

\begin{tabular}{|c|c|c|c|c|c|c|c|}
\hline Année & Sexe & $\begin{array}{l}\text { Classe } \\
\text { sociale }^{\dagger}\end{array}$ & $\begin{array}{l}\text { Éducation } \\
\text { en français } \\
(\%)\end{array}$ & $\begin{array}{l}\text { Exposition } \\
\text { à la radio et } \\
\text { à la télévision } \\
\text { française }\end{array}$ & $\begin{array}{l}\text { Séjour en } \\
\text { milieu } \\
\text { francophone }\end{array}$ & $\begin{array}{l}\text { Séjour dans } \\
\text { une famille } \\
\text { francophone }\end{array}$ & $\begin{array}{l}\text { Langues } \\
\text { parlées } \\
\text { au foyer }\end{array}$ \\
\hline $\begin{array}{l}9 \\
(\mathrm{~N}=2 \mathrm{I})\end{array}$ & $\begin{array}{l}\mathrm{f} \\
(\mathrm{N}=\mathrm{I} 3) \\
\mathrm{m} \\
(\mathrm{N}=8)\end{array}$ & $\begin{array}{l}\text { petite et } \\
\text { moyenne } \\
\text { bourgeoisie } \\
(\mathrm{N}=\mathrm{IO}) \\
\text { ouvrière } \\
\text { supérieure } \\
(\mathrm{N}=9)\end{array}$ & $\begin{array}{l}\mathrm{O}-25 \\
(\mathrm{~N}=2) \\
26-37 \\
(\mathrm{~N}=\mathrm{I} 4) \\
38-\mathrm{IOO} \\
(\mathrm{N}=5)\end{array}$ & $\begin{array}{l}\text { jamais } \\
(\mathrm{N}=\mathrm{I} 6) \\
\text { parfois } \\
(\mathrm{N}=5)\end{array}$ & $\begin{array}{l}\mathrm{oh}-\mathrm{Ij} \\
(\mathrm{N}=8) \\
\mathrm{I}-7 \mathrm{j} \\
(\mathrm{N}=6) \\
7 \mathrm{j}-3 \mathrm{~s} \\
(\mathrm{~N}=6) \\
3 \mathrm{~s}^{+} \\
(\mathrm{N}=\mathrm{I})\end{array}$ & $\begin{array}{l}\text { oh } \\
(\mathrm{N}=\mathrm{I} 5) \\
\mathrm{I}-\mathrm{I} 3 \mathrm{j} \\
(\mathrm{N}=5) \\
2 \mathrm{~s}^{+} \\
(\mathrm{N}=\mathrm{I})\end{array}$ & $\begin{array}{l}\text { anglais } \\
(\mathrm{N}=\mathrm{IO}) \\
\text { romanes } \\
(\mathrm{N}=4) \\
\text { autres } \\
(\mathrm{N}=7)\end{array}$ \\
\hline $\begin{array}{l}\mathrm{I} 2 \\
(\mathrm{~N}=20)\end{array}$ & $\begin{array}{l}\mathrm{f} \\
(\mathrm{N}=\mathrm{I} 7) \\
\mathrm{m} \\
(\mathrm{N}=3)\end{array}$ & $\begin{array}{l}\text { moyenne } \\
(\mathrm{N}=\mathrm{I} 4) \\
\text { moyenne } \\
\text { inférieure } \\
(\mathrm{N}=6)\end{array}$ & $\begin{array}{l}\mathrm{O}-25 \\
(\mathrm{~N}=6) \\
26-37 \\
(\mathrm{~N}=\mathrm{I} 3) \\
38-\mathrm{IOO} \\
(\mathrm{N}=\mathrm{I})\end{array}$ & $\begin{array}{l}\text { jamais } \\
(\mathrm{N}=9) \\
\text { parfois } \\
(\mathrm{N}=\mathrm{II})\end{array}$ & $\begin{array}{l}\text { oh }-\mathrm{rj} \\
(\mathrm{N}=4) \\
\mathrm{I}-7 \mathrm{j} \\
(\mathrm{N}=3) \\
7 \mathrm{j}-3 \mathrm{~s} \\
(\mathrm{~N}=9) \\
3 \mathrm{~s}^{+} \\
(\mathrm{N}=4)\end{array}$ & $\begin{array}{l}\mathrm{oh} \\
(\mathrm{N}=\mathrm{I} 2) \\
\mathrm{I}-\mathrm{I} 3 \mathrm{j} \\
(\mathrm{N}=\mathrm{I}) \\
2 \mathrm{~s}^{+} \\
(\mathrm{N}=7)\end{array}$ & $\begin{array}{l}\text { anglais } \\
(\mathrm{N}=\mathrm{Io}) \\
\text { romanes } \\
(\mathrm{N}=4) \\
\text { autres } \\
(\mathrm{N}=6)\end{array}$ \\
\hline $\begin{array}{l}\text { Total } \\
\mathrm{N}=4 \mathrm{I}\end{array}$ & $\begin{array}{l}\mathrm{f} \\
\mathrm{N}=30 \\
\mathrm{~m} \\
\mathrm{~N}=\mathrm{I} \mathrm{I}\end{array}$ & $\begin{array}{l}\text { moyenne } \\
\mathrm{N}=24 \\
\text { moy. inf. } \\
\mathrm{N}=\mathrm{I} 5\end{array}$ & $\begin{array}{l}0-25 \\
N=8 \\
26-37 \\
N=27 \\
38-100 \\
N=6\end{array}$ & $\begin{array}{l}\text { jamais } \\
\mathrm{N}=25 \\
\text { parfois } \\
\mathrm{N}=\mathrm{I} 6\end{array}$ & $\begin{array}{l}\mathrm{oh}-\mathrm{ij} N=\mathrm{N} 2 \\
\mathrm{I}-7 \mathrm{j} N=9 \\
7 \mathrm{j}-3 \mathrm{~s} N=\mathrm{I} 5 \\
3 \mathrm{~s}^{+} \mathrm{N}=5\end{array}$ & $\begin{array}{l}\mathrm{oh} \\
\mathrm{N}=27 \\
\mathrm{I}-\mathrm{I} 3 \mathrm{j} \\
\mathrm{N}=6 \\
2 \mathrm{~s}^{+} \\
\mathrm{N}=8\end{array}$ & $\begin{array}{l}\text { anglais } \\
\mathrm{N}=2 \mathrm{O} \\
\text { romanes } \\
\mathrm{N}=8 \\
\text { autres } \\
\mathrm{N}=\mathrm{I} 3\end{array}$ \\
\hline
\end{tabular}

${ }^{\dagger}$ Deux élèves n’ont pas fourni de données suffisamment précises pour que l’on puisse déterminer l'appartenance socio-professionnelle de leurs parents. 
francophone et $4 \mathrm{I} \%$ d'entre eux ont passé deux semaines ou plus dans une famille francophone. Par contraste, seulement 33\% des élèves de $9^{\text {ème }}$ année ont séjourné plus d'une semaine dans un milieu francophone et $5 \%$ d'entre eux ont passé deux semaines ou plus dans une famille francophone. Les séjours dans une famille ou dans un milieu francophone ont eu lieu en grande majorité au Québec.

Chacun des 4I élèves a été interviewé par une locutrice native du français pendant environ une heure. Les entrevues ont été enregistrées sur magnétophone. Elles étaient semi-dirigées et portaient sur un ensemble de sujets variés, conçu pour amener les locuteurs à produire, en situation d'entrevue, des registres plus ou moins formels ou informels du français dépendant du sujet abordé (p. ex. un «bon tour» joué à un des professeurs de l'élève, vs l'importance de la religion dans la vie de l'élève). Les entrevues ont été transcrites dans leur totalité en utilisant le système orthographique suppléé, le cas échéant, par une transcription phonétique de certains segments. Ces transcriptions d'entrevue constituent le corpus de français parlé sur lequel repose notre étude.

\section{LA VARIABLE}

La variable sociostylistique à laquelle la présente étude est consacrée inclut l'ensemble des formes verbales qui expriment la notion de futur. Dans les deux sections suivantes nous allons résumer les résultats des études qui ont examiné l'emploi de cette variable par les locuteurs Li du français québécois et présenter des données sur son emploi par les enseignants d'immersion en salle de classe et dans les manuels de français. Ces résultats nous serviront de base de comparaison pour notre analyse de l'emploi de cette variable par les élèves d'immersion.

\section{I Les variantes du futur en français québécois}

Trois études ont examiné l'emploi des variantes du futur en français québécois et ontarien (Deshaies et Laforge, I98 I; Emirkanian et Sankoff, I985 et Poplack et Turpin, I999). Ces études ont identifié trois variantes: a) le futur fléchi; b) le futur périphrastique; et iii) le présent de l'indicatif. Nous présentons, ci-dessous, des exemples qui illustrent l'emploi de ces variantes, qui sont tirés du corpus de Poplack (québécois et franco-ontarien). ${ }^{3}$

\section{Le futur périphrastique (aller + verbe + infinitif)}

I. Bien demain, tu vas aller au bingo

Le futur fléchi (verbe $+-r a i,-r a s,-r a$, etc.)

2. . . on ira à messe demain.

\section{Le présent de l'indicatif}

3. ... j’y vas demain matin.

${ }^{3}$ Le corpus de Poplack (voir Poplack, 1989) a été construit en suivant la même méthodologie labovienne que nous avons employée dans la construction de notre propre corpus. 
Les études mentionnées plus haut ont dégagé les tendances suivantes. Le futur périphrastique est, de loin, la variante la plus fréquente (p. ex. elle a un taux de fréquence de presque $75 \%$ dans le corpus utilisé par Poplack et Turpin, I999, cf. tableau 4). Dans la plupart des contextes où, selon les grammairiens, on devrait s'attendre à trouver une tendance à employer le futur fléchi, les locuteurs du français québécois emploient le futur périphrastique aussi souvent (ou plus souvent) que le futur fléchi (p. ex. contextes hypothétiques, contextes temporels éloignés, etc.). Poplack et Turpin (I999: I60) résument cette tendance ainsi: 'en français canadien, le futur périphrastique ne possède pas de marque sémantique distinctive, il fonctionne plutôt comme un futur générique de base' (notre traduction).

Tableau 4. Fréquence des variantes du futur dans le parler des locuteurs L1 (Ottawa) Hull) et des enseignants d'immersion et dans les manuels de français

\begin{tabular}{|c|c|c|c|c|c|c|}
\hline \multirow[b]{2}{*}{ Corpus } & \multicolumn{2}{|c|}{ Futur fléchi } & \multicolumn{2}{|c|}{$\begin{array}{l}\text { Futur } \\
\text { périphrastique }\end{array}$} & \multicolumn{2}{|l|}{$\begin{array}{l}\text { Présent de } \\
\text { l'indicatif }\end{array}$} \\
\hline & \# & $\%$ & \# & $\%$ & $\#$ & $\%$ \\
\hline $\begin{array}{l}\text { Locuteurs LI } \\
\text { (Corpus Poplack) }\end{array}$ & $725 / 3594$ & 20 & $2627 / 3594$ & 73 & $242 / 3594$ & $\overline{7}$ \\
\hline $\begin{array}{l}\text { Enseignants } \\
\text { d'immersion }\end{array}$ & $98 / 547$ & I 8 & $434 / 547$ & 79 & I $5 / 547$ & 3 \\
\hline Manuels (écrit) & $400 / 420$ & 95,3 & $20 / 420$ & 4,7 & $0 / 420$ & o \\
\hline Manuels («oral») & $33 / 47$ & 70,3 & $\mathrm{I} 4 / 47$ & 29,7 & $\mathrm{O} / 47$ & $\mathrm{o}$ \\
\hline
\end{tabular}

Le futur simple, quant à lui, est fortement associé aux contextes suivants: i) les phrases négatives; ii) les énoncés plus ou moins figés, p. ex. les commandements de la Bible, les prédictions; iii) les énoncés où le locuteur emploie le pronom d'adresse vous plutôt que $t u$. Pour ce qui est du présent de l'indicatif, Poplack et Turpin (I999) signalent qu'il est surtout employé dans les contextes où l'action est localisée dans le futur à l'aide d'un adverbe de temps. En d'autres termes, en l'absence d'un tel adverbe, le présent de l'indicatif peut difficilement exprimer à lui seul la notion de futur. Chose intéressante, la plupart de ces tendances ont échappé à l'attention des grammairiens et des locuteurs du français.

Pour ce qui est de l'effet des facteurs sociaux, on a trouvé que le futur fléchi est moins fréquent dans le parler des jeunes locuteurs que dans celui des locuteurs âgés. Selon Poplack et Turpin, ce résultat, ainsi que la spécialisation contextuelle et la faible fréquence du futur fléchi, suggèrent que ce temps est en phase de disparition dans le discours informel, changement qui rappelle ce qui est arrivé au passé simple et ce qui est en train d'arriver au subjonctif. Quant à l'appartenance socioprofessionnelle des locuteurs, elle n'exerce pas d'effet significatif sur le choix des variantes. Ce dernier résultat suggère qu'en français canadien parlé, la variable qui nous intéresse fonctionne plus comme un marqueur de style que comme un indicateur d'appartenance socio-économique. 
Par contraste, en ce qui a trait à l'usage des variantes du futur en français québécois écrit, Lesage et Gagnon (I992) ont trouvé que le futur fléchi domine largement sur le futur périphrastique et non l'inverse. Ce résultat permet de mieux comprendre pourquoi dans le corpus utilisé par Poplack et Turpin (I999) le futur fléchi est associé au discours plus ou moins formulaique et formel.

\subsection{Les variantes du futur dans le français des enseignants et des manuels}

Comme le montre le tableau 4, en salle de classe, les enseignants d'immersion utilisent les mêmes variantes du futur que les locuteurs LI en situation d'entrevue. De plus, il est remarquable qu'il n'y a pas de grosses différences de fréquence d'occurrence entre le parler de ces locuteurs et celui des enseignants. Si ces derniers font un emploi un peu plus fréquent du futur fléchi, ils utilisent par contre le futur périphrastique presque aussi souvent que les locuteurs LI. Le fait que la variable est dépourvue de marque sociale explique peut-être en partie ce résultat. Toutefois, compte tenu du fait que le futur fléchi est associé au discours plus formel et est très largement dominant dans les manuels (résultat conforme à l'étude de Lesage et Gagnon, I992), on se serait attendu, en ce qui concerne cette variante, à ce que la différence de fréquence entre les locuteurs LI et les enseignants soit nettement plus prononcée. C'est ce que nous avons trouvé, par exemple, dans le cas de l'effacement de la particule négative ne: phénomène rarissime dans les manuels (y compris les dialogues et autres représentations de l'oral), peu fréquent dans le discours des enseignants d'immersion en classe et quasi-catégorique dans le discours des locuteurs Li (Rehner et Mougeon, I999; Rehner, Mougeon et Nadasdi, à paraître).

En ce qui concerne les manuels de français, les différences de fréquence intervariantes sont plus spectaculaires. En effet, on constate que le présent est inexistant et que le futur fléchi est la variante largement dominante et ce même dans les textes qui sont censés représenter la langue parlée (on remarque cependant que dans ces derniers textes la fréquence du futur périphrastique est sensiblement plus élevée).

Dans le cas de variation qui nous intéresse ici, la comparaison des données sur la langue des enseignants et celle des manuels (les deux principales sources d'exposition au français pour les élèves d'immersion) est particulièrement intéressante. En effet, elle révèle que les élèves sont confrontés à deux normes largement divergentes. Nous avons déjà observé ce genre de situation «cornélienne» dans une étude antérieure (Rehner, Mougeon et Nadasdi, à paraitre) consacrée à la variation on vs nous. Les enseignants emploient massivement on (taux de fréquence: $83 \%$ ) et les manuels privilégient nous (taux de fréquence: $74 \%$ ). Dans cette dernière étude on a trouvé que les élèves d'immersion utilisent la variante on à un taux de fréquence de $56 \%$, c'est-à-dire à un niveau de fréquence intermédiaire entre celui des enseignants et des manuels. Nous avons attribué ce résultat principalement au fait que les élèves sont en quelque sorte pris entre la norme des enseignants et celle des manuels. Ceci dit nous avons aussi mentionné le fait que notre échantillon inclut $25 \%$ d'élèves qui parlent l'italien ou l'espagnol (deux langues qui ne possèdent pas de pronoms 
semblables à on mais des pronoms qui ressemblent à nous, à savoir noi et nosotros). Comme ces élèves emploient on à un taux de fréquence de $15 \%$ seulement, il est possible que cela ait diminué la fréquence moyenne de on dans notre corpus!

\subsection{Hypothèses sur la fréquence des variantes dans le parler des élèves}

À la lumière des résultats de notre étude de la variable on vs nous, nous nous attendions à trouver à nouveau que les élèves soient divisés entre les deux normes et donc qu'ils fassent un emploi assez fréquent du futur fléchi, tendance qui pourrait se voir renforcée dans le cas des élèves qui parlent italien ou espagnol, car ces deux langues, contrairement à l'anglais, possèdent un futur fléchi. Toutefois, nous nous attendions aussi à trouver que les élèves emploient les deux autres variantes aussi souvent (sinon plus souvent) que le futur fléchi et ce pour plusieurs raisons. Dans son étude sur l'acquisition des formes verbales par les élèves d'immersion Harley (I992) a constaté que les élèves avaient des difficultés considérables à maitriser les formes verbales du conditionnel (formes synthétiques qui n'ont pas d'équivalents en anglais). Or, dans le cas de variation qui nous intéresse, si l'anglais n'a pas de futur fléchi, il possède deux variantes du futur qui sont similaires aux deux autres variantes du français: i) la construction périphrastique gonna + verbe $^{4}$ ) et ii) le présent de l'indicatif. En d'autres termes, on pourrait s'attendre à ce que les élèves convergent naturellement sur ces deux alternatives plus conformes à la morphosyntaxe de l'anglais. La tendance à favoriser les variantes périphrastique et présent nous semble d'autant plus probable étant donné les recherches qui démontrent que les apprenants préferent les structures analytiques aux structures synthétiques (cf. Meisel, I983; Andersen, I990; Van Patten, I990). Notons finalement que ce même résultat se retrouve chez les enfants francophones. Plus précisément, à l'âge de 26 mois, l'enfant étudié par Sabeau-Jouannet (I977) a produit I4 occurrences de la variante périphrastique et aucune occurrence du futur fléchi; à l'âge de 3 ans, ce même enfant a produit 40 périphrastiques et 12 fléchis. Tous ces faits (la structure similaire de l'anglais, les tendances développementales déjà documentées) nous poussent à prévoir que le futur fléchi sera relativement rare dans notre corpus.

\section{LES VARIANTES DU FUTUR DANS LE FRANÇAIS PARLÉ DES ÉLÈVES D'IMMERSION}

\section{I Fréquence des variantes}

On peut remarquer, tout d'abord, que dans le parler des élèves on observe les trois variantes que l'on retrouve en français canadien parlé, mais aussi des formes non

${ }^{4}$ Cette similarité est soulignée par Harley (1982: I03) qui note: 'Given not only the lexical relationship between go and aller but also the formal analytic and functional similarity with English be + going to + infinitive, it may be assumed that the French periphrastic future is more readily acquired by English-speaking learners than the future simple'. 
natives. L'attestation de ces dernières formes est conforme à une tendance générale amplement attestée dans la recherche sur la compétence linguistique productive des élèves d'immersion. Ceux-ci ne maîtrisent pas la plupart des aspects complexes de la morphologie ou du lexique du français au même niveau que les locuteurs natifs du français.

Voici des exemples de ces variantes et d'une des formes non natives, tirés de notre corpus de français d'immersion.

\section{Le futur périphrastique (aller + verbe + infinitif $)$}

4. nous allons faire une fête.

Le futur fléchi (verbe $+-r a i,-r a s,-r a$, etc.)

5. l'air sera très difficile à respirer.

\section{Le présent de l'indicatif}

6. à Noël . . . cette année on reste à la maison.

Les formes non natives (formes comportant une erreur de personne, de nombre de temps, de mode, etc.)

7. à l'avenir . . tout le monde ser[E] parfait.

Si l'on fait abstraction des formes non natives, dont la fréquence n'est certes pas négligeable (cf. tableau s) et sur lesquelles nous reviendrons plus bas, on constate que les élèves font un usage modéré du futur fléchi et du présent (I I \% chacun) et inversement qu'ils montrent une nette préférence pour le futur périphrastique (78\%). En d'autres termes, contrairement à notre recherche sur l'acquisition de la variation on vs nous, la divergence de norme entre le parler des enseignants (prépondérance du futur périphrastique) et des manuels (prédominance du futur fléchi) et la présence de futurs fléchis en italien et espagnol n'ont pas joué en la faveur de la variante plus formelle (le futur fléchi). ${ }^{5}$ En effet, on constate que les élèves d'immersion utilisent majoritairement le futur périphrastique, forme qu'on

Tableau 5. Fréquence des quatre variantes du futur dans le corpus des élèves d'immersion

\begin{tabular}{lccc}
\hline \hline Variante & Nombre & Pourcentage & $\begin{array}{l}\text { \% sans les formes } \\
\text { non natives }\end{array}$ \\
\hline Futur périphrastique & 273 & 67 & 78 \\
Future fléchi & 39 & IO & I I \\
Présent de l'indicatif & 40 & IO & I I \\
Formes verbales non natives & 55 & I 4 & IOO \\
TOTAL & 407 & IOO & \\
\hline \hline
\end{tabular}

${ }^{5}$ Nous allons voir plus bas que, contrairement à notre attente, les élèves qui parlent l'une ou l'autre de ces deux langues romanes au foyer, ne se distinguent pas par un emploi plus fréquent du futur fléchi. 
trouve en anglais. Il est du reste intéressant de constater que cet effet de convergence n'est pas limité au futur périphrastique puisque les élèves emploient le présent plus souvent que les enseignants et les locuteurs natifs ( I I \% vs $3 \%$ et $7 \%$ ).

\subsection{Contraintes linguistiques}

On a vu plus haut que dans nos recherches antérieures les élèves d'immersion ont une maitrise imparfaite des contraintes linguistiques observées dans le discours des locuteurs Li. Or, on vient de voir qu'au niveau de la fréquence des variantes, le parler des élèves n'est pas trop éloigné de celui des locuteurs LI et des enseignants d'immersion. Se pourrait-il donc que, contrairement à la tendance générale, les élèves aient pour ce qui est de la variable à l'étude, une maitrise des contraintes comparable à celle des locuteurs LI?

Notre étude de l'effet des contraintes linguistiques sur l'emploi des trois variantes du futur a été réalisée à l'aide du logiciel GoldVarbII (Rand et Sankoff, I990). Ce logiciel effectue une analyse factorielle à régression multiple qui permet d'identifier les facteurs linguistiques ou extra-linguistiques qui sont corrélés avec l'emploi des variantes. L'analyse factorielle a porté sur dix facteurs linguistiques qui ont été examinés dans les études sur l'emploi des variantes du futur par les locuteurs Li. Les résultats de cette analyse factorielle ainsi que les facteurs linguistiques identifiés par les études consacrées au français parlé des locuteurs LI sont indiqués respectivement dans les tableaux 6 et 7 .

Tableau 6. Facteurs linguistiques corrélés à l'emploi des variantes du futur dans le parler des élèves d'immersion

\begin{tabular}{llll}
\hline \hline Facteurs linguistiques & Fléchi & Périphrastique & Présent \\
\hline Adverbe temporel & \multicolumn{2}{c}{ Effets des facteurs ${ }^{\dagger}$} & \\
$\quad$ Spécifique & NS & .333 & $.7 \mathrm{I} 4$ \\
Non spécifique & NS & .396 & .637 \\
Pas d'adverbe & NS & .655 & .300 \\
Distance temporelle de l'action & NS & NS & NS \\
Négation de la phrase & NS & NS & NS \\
Personne du verbe & NS & NS & NS \\
Contingence de l'action & NS & NS & NS \\
Certitude de l'action & NS & NS & NS \\
\hline \hline
\end{tabular}

NS $=$ Non significatif.

†L'effet d'un facteur provient d'une analyse à régression multiple fournie par GoldVarbII. L'effet de facteur représente la contribution relative d'un facteur donné par rapport aux autres facteurs du même groupe. Un effet supérieur à .500 favorise l'application de la règle, alors qu'un effet inférieur à .500 la défavorise. 
Tableau 7. Facteurs linguistiques corrélés à l'emploi des variantes du futur dans le parler des locuteurs L1 (corpus Poplack)

\begin{tabular}{|c|c|c|c|}
\hline Facteurs linguistiques & Fléchi & Périphrastique & Présent \\
\hline Adverbe temporel & \multicolumn{3}{|c|}{ Effets des facteurs } \\
\hline Spécifique & .37 & .23 & .78 \\
\hline Non spécifique & .85 & .19 & .58 \\
\hline Pas d'adverbe & .47 & .56 & .46 \\
\hline \multicolumn{4}{|c|}{ Distance temporelle de l'action } \\
\hline Proximale & .52 & .56 & .44 \\
\hline Distale & .48 & .43 & .57 \\
\hline \multicolumn{4}{|l|}{ Négation de la phrase } \\
\hline Négative & .99 & $.0 \mathrm{I}$ & NS \\
\hline Affirmative & .36 & .65 & NS \\
\hline \multicolumn{4}{|l|}{ Personne du verbe } \\
\hline Pronom d'adresse Vous & $.8 \mathrm{I}$ & .22 & NS \\
\hline Autres sujets & .49 & $.5 \mathrm{I}$ & NS \\
\hline \multicolumn{4}{|l|}{ Contingence de l'action } \\
\hline Contingent & $.5 \mathrm{I}$ & NS & NS \\
\hline Non-contingent & .45 & NS & NS \\
\hline \multicolumn{4}{|l|}{ Imminence } \\
\hline Imminent & NS & NS & .54 \\
\hline Non imminent & NS & NS & .13 \\
\hline
\end{tabular}

NS $=$ Non significatif.

La seule contrainte linguistique qui est observée par les élèves d'immersion est la présence d'un adverbe temporel qui localise l'action dans le futur. Notre évaluation de l'effet de ce facteur repose sur une distinction ternaire, présence d'un adverbe temporel spécifique, vs présence d'un adverbe temporel non spécifique, vs absence d'un adverbe temporel, cf. les exemples ci-dessous:

\section{Adverbe spécifique}

8. l'été qui vient je vais chez Ireland.

\section{Adverbe non spécifique}

9. ça va être très important dans le futur.

\section{Pas d'adverbe}

Io. je pense que ça va être moi.

À l'instar de Poplack et Turpin (I999), nous attribuons l'effet positif de la présence d'un adverbe de temps sur l'emploi du présent de l'indicatif à l'ambiguïté de la référence temporelle de ce temps. Employé sans adverbe de temps qui localise l'action dans le futur, le présent de l'indicatif tend à exprimer une action inachevée ou habituelle. Lorsqu'un tel adverbe est présent, l'ambiguïté est levée et le verbe au présent prend une valeur de futur.

Parmi les contraintes linguistiques qui sont observées par les locuteurs Li et que nos élèves d'immersion ne respectent pas, la contrainte relative aux phrases négatives mérite une mention particulière. En effet, dans le parler des locuteurs du français 
québécois, les phrases négatives sont étroitement associées à l'emploi du futur fléchi (cf. Deshaies et Laforge, I98 I; Emirkanian et Sankoff, I985; et Poplack et Turpin, I999). En fait, dans l'étude de Poplack et Turpin, c'est surtout dans ce contexte que les locuteurs emploient le futur fléchi (cf. le caractère remarquablement élevé de l'effet de ce contexte: 99 trouvé dans l'étude de Poplack et Turpin, I999, voir le tableau 7 ci dessus). ${ }^{6}$ Finalement on peut remarquer que dans le cas des contraintes associées à l'opposition phrase négative vs affirmative et présence vs absence d'un adverbe de temps plus ou moins spécifique, et vous vs les autres sujets, on remarque que dans le parler des locuteurs Li les différences d'effet interfactoriel sont prononcées (cf. tableau 7). Par contraste, dans le cas des autres contraintes les différences d'effet sont plus ténues, et il n'est donc peut-être pas étonnant que les élèves d'immersion n'observent pas ces contraintes linguistiques moins marquées. En bref, notre étude de l'acquisition des contraintes linguistiques de la variable futur confirme la tendance générale dégagée par nos études antérieures et elles suggèrent que si les élèves emploient les variantes à des taux de fréquences qui ne sont pas trop éloignés de ceux des locuteurs Li, c'est peut-être plus à cause d'une combinaison de facteurs: a) les enseignants en font autant; b) l'anglais entraîne un phénomène de convergence; c) il existe une tendance naturelle à préférer les structures analytiques.

\section{FACTEURS EXTERNES}

Dans notre analyse factorielle de l'effet des facteurs externes sur l'emploi des variantes du futur, nous avons inclus les paramètres sociaux traditionnels (classe sociale et sexe), le niveau de scolarité et plusieurs facteurs qui sont propres à nos élèves: la/les langue(s) parlée(s) à la maison, la durée des séjours dans un environnement francophone, des séjours chez une famille francophone et la fréquence de l'écoute des média francophones, autant de facteurs qui se sont avérés influencer l'acquisition de la variation sociostylistique par les élèves inclus dans notre corpus (cf. section 3 ).

Les facteurs externes qui ont été retenus par le logiciel GoldVarb sont présentés dans le tableau 8. Parmi tous les facteurs sociaux qui sont corrélés avec l'emploi des variantes du futur, on peut mentionner en premier le paramètre 'durée des séjours dans un environnement francophone'. Nous avons mesuré l'effet de ce facteur à l'aide d'une échelle à quatre points (o: un jour ou moins d'un jour; $\mathrm{I}$ : deux à sept jours; 2: huit jours à trois semaines; 3 : plus de trois semaines). Comme le montre le tableau 8 , il y a une corrélation linéaire parfaite entre ce facteur et la fréquence d'emploi du futur fléchi et du futur périphrastique: plus les séjours en milieu francophone ont été longs plus la fréquence d'emploi du futur périphrastique est élevée et inversement moins la fréquence d'emploi du futur fléchi est élevée. Ceci

${ }^{6}$ Toutefois, ce n'est pas le cas pour toute variété de français canadien. En effet, en français acadien les phrases négatives n'exercent pas d'effet particulier sur le futur fléchi (King et Nadasdi, à paraître). 
Tableau 8. Effet des facteurs externes sur l'emploi des variantes du futur par les élèves d'immersion

\begin{tabular}{|c|c|c|c|c|c|c|c|c|c|}
\hline \multirow{2}{*}{$\begin{array}{l}\text { Facteurs } \\
\text { externes }\end{array}$} & \multicolumn{3}{|c|}{ Futur fléchi } & \multicolumn{3}{|c|}{ Futur périphrastique } & \multicolumn{3}{|c|}{ Présent de l'indicatif } \\
\hline & $\mathrm{N}$ & $\%$ & Effets & $\mathrm{N}$ & $\%$ & Effets & $\mathrm{N}$ & $\%$ & Effets \\
\hline \multicolumn{10}{|c|}{$\overline{\text { Niveau de scolarité }}$} \\
\hline $9^{\text {ème }}$ & $29 / \mathrm{I} 83$ & I6 & .628 & I 3 I / I 83 & 72 & $.4 \mathrm{I} 5$ & $23 / 183$ & I 3 & NS \\
\hline $12^{\text {ème }}$ & IO/I 69 & 6 & $.36 \mathrm{I}$ & I $42 /$ I 69 & 84 & .592 & $\mathrm{I} 7 / \mathrm{I} 69$ & IO & NS \\
\hline \multicolumn{10}{|c|}{ Milieu francophone } \\
\hline o & $22 / 93$ & 24 & .774 & $57 / 93$ & 6I & .285 & I $4 / 93$ & I 5 & .587 \\
\hline I & $7 / 63$ & 22 & .580 & $43 / 63$ & 68 & .310 & I3/63 & $2 \mathrm{I}$ & .740 \\
\hline 2 & $9 / \mathrm{I} 35$ & 7 & $.44 \mathrm{I}$ & I $15 /$ I 35 & 85 & .600 & II / I 35 & 8 & $.4 \mathrm{I} 8$ \\
\hline 3 & $\mathrm{I} / 6 \mathrm{I}$ & 2 & .156 & $58 / 6 \mathrm{I}$ & 95 & .790 & $2 / 6 \mathrm{I}$ & 3 & .293 \\
\hline \multicolumn{10}{|l|}{ Sexe } \\
\hline Masculin & $2 / 76$ & 3 & .203 & $62 / 76$ & 82 & NS & $\mathrm{I} 2 / 76$ & I6 & NS \\
\hline Féminin & $37 / 276$ & I 3 & .593 & $2 \mathrm{II} / 276$ & 76 & NS & $28 / 276$ & IO & NS \\
\hline \multicolumn{10}{|c|}{ Langue(s) du foyer } \\
\hline Anglais & $28 /$ I 86 & I 5 & $.62 \mathrm{I}$ & I $34 /$ I 86 & 72 & $.4 \mathrm{I} 3$ & $24 /$ I 86 & I 3 & NS \\
\hline Romane & $7 / 63$ & I I & .536 & $46 / 63$ & 73 & $.4 \mathrm{IO}$ & Io/63 & I6 & NS \\
\hline Autres & $7 / \mathrm{IO} 3$ & 4 & .273 & 93/IO3 & 90 & .703 & $6 / 193$ & 6 & NS \\
\hline
\end{tabular}

dit, si on regarde de près les pourcentages de fréquence et les effets factoriels calculés par GoldVarb, on peut se demander si l'influence de ce facteur est entièrement souhaitable. En effet, on constate que plus la durée du séjour en milieu francophone est longue plus la fréquence d'emploi du futur périphrastique et du futur fléchi s'éloigne de la norme des locuteurs Li, à tel point que dans le parler des locuteurs qui ont séjourné le plus longtemps dans un milieu francophone, le futur périphrastique a presque totalement évincé les deux autres variantes! Ce résultat inattendu est peutêtre dû à la grande fréquence du futur périphrastique dans le parler des locuteurs Li. Plus les élèves ont des contacts avec ces locuteurs, plus cela renforcerait leur tendance naturelle à employer la variante périphrastique. ${ }^{7}$ Quoi qu'il en soit, il faudrait se garder de conclure pour autant que l'influence du facteur durée du séjour en milieu francophone est essentiellement négative. En effet, on ne doit pas perdre de vue, que les élèves qui n'ont pas effectué de tels séjours, ou dont les séjours ont été courts (valeurs o et I sur l'échelle de durée), emploient un peu plus souvent le futur fléchi que les locuteurs Li. Par ailleurs, les élèves ayant effectué les séjours les plus longs (valeurs 3 et 4 ) sur-utilisent la variante qui, pour reprendre l'expression de Poplack et Turpin (I999), est le «futur de base non marqué», c'est-à-dire le futur 'passe partout' que les locuteurs Li emploient couramment.

L'analyse factorielle a aussi conclu que le niveau de scolarité des élèves était lui aussi corrélé à l'emploi des variantes. Toutefois, nous avons vu plus haut dans

${ }^{7}$ Reste à savoir si des séjours prolongés en milieu francophone se traduiraient par un réajustement à la baisse de la fréquence d'emploi du futur périphrastique! 
la section où nous avons présenté notre échantillon d'élèves, qu'il y a une forte interaction entre le facteur durée des séjours dans un milieu ou dans une famille francophone et le niveau de scolarité, les élèves de $9^{\text {ème }}$ année ayant effectué beaucoup moins de tels séjours que les élèves de $\mathrm{I} 2^{\text {ème }}$ année. Il est donc probable que les différences de fréquence d'emploi du futur fléchi et du futur périphrastique en fonction du niveau de scolarité reflètent en partie l'influence du facteur durée du séjour en milieu francophone.

En bref, les résultats relatifs à l'effet des paramètres séjour en milieu francophone et niveau de scolarité confirment les résultats de nos recherches antérieures: les contacts extra-scolaires avec les locuteurs LI favorisent l'acquisition des variantes qui font partie de l'usage courant de ces locuteurs.

Le tableau 8 montre aussi que le sexe exerce un effet significatif sur l'emploi des variantes qui se traduit par un emploi nettement plus fréquent du futur fléchi par les filles que par les garçons. Nous avons déjà observé cette tendance dans nos recherches antérieures (cf. section 3). Dans la recherche sociolinguistique sur la variation dans les langues Li, on a souvent constaté le même type de tendance, les femmes font un usage plus fréquent des variantes standard que les hommes (Labov, I990). En d'autres termes, on peut faire l'hypothèse que les élèves d'immersion du sexe féminin sont plus sensibles que les élèves du sexe masculin au traitement préférentiel accordé au futur fléchi par les manuels de français et que ceci les conduit à préférer cette variante.

Finalement, l'analyse factorielle effectuée par GoldVarb a aussi conclu que la langue parlée au foyer est corrélée avec l'emploi des variantes: les élèves qui parlent une langue autre que l'anglais ou une langue romane emploient plus souvent le futur périphrastique. Ceci dit, il nous est difficile de proposer une interprétation pour cette corrélation. Ces élèves parlent un large éventail de langues différentes dont le futur est plus ou moins proche ou différent du futur du français. On ne s'attendait donc pas à ce que ces langues exercent une influence uniforme sur l'emploi des variantes du futur. Par ailleurs, il est remarquable que les élèves qui parlent une langue romane n'emploient pas plus souvent le futur fléchi que les élèves qui ne parlent que l'anglais au foyer. En d'autres termes la présence de futurs fléchis dans les langues romanes ne semble pas exercer un effet particulièrement favorable à l'emploi du futur fléchi français. Ce résultat pourrait peut-être s'attribuer au fait que même si le futur fléchi s'emploie dans d'autres langues romanes, ce n'est pas la forme dominante.

\section{LES VARIANTES NON NATIVES}

On a vu plus haut (cf. tableau 5 et exemple 7) que les élèves d'immersion ont recours à des formes non natives pour exprimer la notion de futur. Ces formes non natives incluent, entre autres, des verbes employés à l'infinitif, au conditionnel présent et à l'imparfait. Puisque ces formes ne constituent pas un ensemble cohérent nous n'avons pas étudié l'influence des facteurs linguistiques sur leur emploi. Toutefois, 
Tableau 9. Effet des facteurs externes sur l'emploi des variantes non natives du futur par les élèves d'immersion

\begin{tabular}{|c|c|c|c|}
\hline Facteurs externes & $\mathrm{N}$ & $\%$ & Effets des facteurs \\
\hline \multicolumn{4}{|l|}{ Niveau de scolarité } \\
\hline 9 & $45 / 228$ & 20 & $.65 \mathrm{I}$ \\
\hline I2 & IO/I 79 & 6 & $.3 \mathrm{II}$ \\
\hline \multicolumn{4}{|c|}{ Instruction en français } \\
\hline I & I I $/ 66$ & I7 & $.58 \mathrm{I}$ \\
\hline 2 & 4I $/ 268$ & I 5 & .556 \\
\hline 3 & $2 / 50$ & 4 & .225 \\
\hline 4 & $\mathrm{I} / 20$ & 5 & .268 \\
\hline \multicolumn{4}{|c|}{ Durée des séjours en milieu francophone } \\
\hline $\mathrm{O}$ & $20 /$ I I 3 & I 8 & $.6 \mathrm{I} 3$ \\
\hline I & I6/79 & 20 & $.65 \mathrm{I}$ \\
\hline 2 & $17 / 152$ & I I & $.48 \mathrm{I}$ \\
\hline 3 & $2 / 63$ & 3 & .195 \\
\hline
\end{tabular}

il nous a paru intéressant d'essayer d'identifier les corrélats externes de l'emploi de ces formes. Les résultats de l'analyse factorielle sont présentés dans le tableau 9.

Trois paramètres externes sont corrélés avec l'emploi des formes non natives: i) le niveau de scolarité, ii) la durée des séjours dans un environnement francophone, et iii) le montant d'éducation en français. Précisons que les différences relatives au troisième paramètre reflètent le fait que certains élèves ont plus ou moins bénéficié d'un surcroît d'instruction en français, ${ }^{8}$ ce pour différentes raisons (p. ex. certains élèves ont été inscrits pendant plusieurs années dans une école de langue française avant leur inscription en programme d'immersion).

Si l'on accepte l'idée que le recours aux formes non natives est plus ou moins symptomatique du fait que nos élèves n'ont pas entièrement maitrisé l'usage du futur, la corrélation avec les trois facteurs mentionnés plus haut constitue un patron cohérent. En effet, on peut constater que les élèves qui utilisent le moins souvent les formes non natives, sont ceux qui ont reçu le plus d'instruction en français, qui ont séjourné le plus longtemps en milieu francophone, ou qui sont à la fin du cycle d'études secondaires. En d'autres termes, plus les élèves ont eu d'occasions d' «apprendre» le français, plus ils ont surmonté les difficultés du maniement du futur, et donc, moins ils ont recours à des formes non natives. Ce résultat rappelle celui de Nadasdi (200I) dans son étude sur le nivellement des marques du pluriel dans les formes verbales du présent de l'indicatif de la $3^{\mathrm{e}}$ personne du pluriel. Dans son étude Nadasdi a pu constater que les élèves qui sont le plus scolarisés en français sont ceux qui conservent le plus les marques de pluralité sur le verbe (voir aussi Bartning, I998).

${ }^{8}$ Nous avons utilisé une échelle à quatre points pour quantifier ces différences: $(\mathrm{I}=3$ à $29 \%$ d'instruction en français; $2=30 \%$ à $39 \% ; 3=40 \%$ à $99 ; 4=100 \%)$. 


\section{LES VARIANTES INFORMELLES DU FUTUR PÉRIPHRASTIQUE}

Comme on a pu le constater dans la section 5.I, hormis la variante du futur fléchi qui tend à être associée au registre soutenu, les deux autres variantes du 'futur' en français ne sont pas dotées d'une forte marque sociostylistique. Or, si l'on se concentre sur l'emploi du futur périphrastique à la I ère personne du singulier, on peut déceler, en français canadien parlé, un phénomène variationnel qui implique trois variantes dont deux sont pourvues d'une forte marque sociostylistique. ${ }^{9}$ Il s'agit de l'alternance entre les variantes je vas vs je vais vs (je)m'as + infinitif (cf. les exemples I I-I 3 sont tirés du corpus de Mougeon et Beniak, ces deux auteurs ayant consacré une étude spéciale à ce phénomène variationnel: Mougeon et Beniak, I99I, ch. 8). Ce cas de variation a été aussi attesté en français québécois, cf. Deshaies et Laforge, (I98I). Dans leur propre étude sur la variable 'futur' Poplack et Turpin (I999) n'ont pas distingué les trois variantes du futur périphrastique à la I ère personne du singulier. Elles les ont traitées dans leur analyse statistique comme une seule variante (futur périphrastique) et ont renvoyé le lecteur à Mougeon et Beniak (I99I) pour des renseignements sur les corrélats sociaux de vais/vas/m'as. ${ }^{10}$

I I. O.K. je vais t'aider.

I2. ben je vas y aller à l'université.

I3. $m$ 'as retourner à Cornwall plus tard.

En plus d'avoir mesuré la fréquence des trois variantes mentionnées plus haut (cf. le tableau Io) Mougeon et Beniak (I99I) ont aussi trouvé que la variante (j)m'as et la variante je vais, étaient corrélées à l'appartenance socioéconomique des locuteurs ( $(j) m$ 'as étant fortement associée au parler des locuteurs des couches sociales les plus basses et je vais étant associée au parler des locuteurs des couches sociales les plus élevées). Ces mêmes auteurs ont aussi trouvé que je vas était, par contraste, dénué de marque sociale.

Avec ce cas de variation, nous avons donc l'occasion de vérifier à nouveau si les élèves d'immersion sur-utilisent les variantes formelles et inversement n'emploient que marginalement (ou pas du tout) les variantes vernaculaires (cf. section 3).

Comme l'indique le tableau io, si on compare l'usage des variantes du futur périphrastique par les élèves d'immersion avec celui des élèves franco-ontariens étudié par Mougeon et Beniak (I99I), on constate que les élèves d'immersion n'emploient pas la variante vernaculaire $m$ 'as, qu'ils emploient la variante je vas nettement moins souvent que les élèves franco-ontariens et qu'ils font un usage beaucoup plus fréquent de je vais que les élèves franco-ontariens.

9 On doit préciser que cette variation ne s'observe qu'à la I ${ }^{\text {ère }}$ personne du singulier. Aux autres personnes, il n'y a qu'une seule forme du futur périphrastique (cf. Mougeon, I996, pour des informations sur les origines géographiques et socio-historiques de la micro-variable vas/vais/m'as).

10 Nous avons procédé de la même façon dans notre étude générale, nous n'avons pas distingué je vais de je vas. Ces deux formes ont été comptées comme des occurrences de la même variante (à savoir le futur périphrastique). 
Tableau Io. Emploi des variantes du futur périphrastique à la $1^{\text {ère }}$ personne du singulier par les élèves d'immersion et les élèves franco-ontariens

\begin{tabular}{|c|c|c|c|c|c|c|c|}
\hline \multirow[b]{2}{*}{ Corpus } & \multicolumn{2}{|c|}{ Je vais } & \multicolumn{2}{|c|}{ Je vas } & \multicolumn{2}{|c|}{ M'as } & \multirow[b]{2}{*}{ TOTAL } \\
\hline & $\mathrm{N}$ & $\%$ & $\mathrm{~N}$ & $\%$ & $\mathrm{~N}$ & $\%$ & \\
\hline Élèves d'immersion & IO4 & 90 & I2 & IO & $\mathrm{o}$ & $\mathrm{o}$ & I I 6 \\
\hline $\begin{array}{l}\text { Élèves franco-ontariens } \\
\text { (Mougeon et Beniak I99I) }\end{array}$ & 52 & $\mathrm{I} 2$ & 266 & 60 & $\mathrm{I} 26$ & 28 & 444 \\
\hline Enseignants d'immersion & 88 & 99 & I & I & $\mathrm{o}$ & $\mathrm{o}$ & 89 \\
\hline Manuels de français & 23 & IOO & $\mathrm{O}$ & $\mathrm{O}$ & $\mathrm{O}$ & $\mathrm{o}$ & 23 \\
\hline
\end{tabular}

Si on examine les données sur l'emploi des variantes du futur périphrastique par les enseignants d'immersion et des manuels de français, on constate que l'usage des élèves d'immersion est très proche de cet emploi. La variante $m$ 'as est absente dans le français des manuels et des enseignants, je vais est très fréquent dans le français des enseignants et catégorique dans les manuels et je vas est marginal dans le français des enseignants et absent dans le français des manuels. En dépit de son caractère socialement non marqué et de sa haute fréquence discursive dans le parler des locuteurs Li en situation d'entrevue enregistrée la variante je vas est exclue de la norme scolaire. Le fait qu'elle n'est pas conforme au français standard international est sans doute à l'origine de cette exclusion. Ceci dit, il est remarquable que dans le parler des élèves d'immersion la variante je vas n'est pas totalement absente et donc on peut s'interroger sur l'origine de cette forme dans leur parler. Deux hypothèses sont envisageables: i) que les élèves l'aient appris par le biais des contacts avec les locuteurs Li; ou bien, ii) que cette forme soit symptomatique de difficultés plus ou moins persistantes à maitriser la forme je vais, dont la morphologie est irrégulière (je vais / vE/ vs tu/il/elle va(s)/va/). Selon cette deuxième hypothèse, l'emploi de je vas serait donc assimilable à une erreur développementale qui se manifesterait par la substitution d'une forme «régulière» à une forme plus difficile car irrégulière (cf. section 3). Pour vérifier ces hypothèses nous avons corrélé l'emploi de je vas avec la durée des séjours dans un milieu francophone.

Comme le révèle le tableau II, il semble bien que la première hypothèse est infirmée et que la deuxième est confirmée. En effet, on constate que la fréquence d'emploi de je vas est en corrélation négative avec la durée des séjours en milieu francophone: plus ces séjours ont été longs, moins la fréquence d'emploi de je vas est élevée! En fait, dans le cas des élèves qui ont séjourné le plus longtemps en milieu francophone, la forme je vas est absente de leur parler. Ces derniers élèves n'auraient donc plus de difficultés avec la forme irrégulière je vais. Toutefois, il est remarquable que leurs contacts avec les locuteurs Li n'ont pas été assez prolongés pour entraîner l'apprentissage de la variante vas, pas plus qu'ils ne leur ont permis d'acquérir la variante vernaculaire m'as. 
Tableau Ir. Emploi de je vais vs je vas en fonction de la durée des séjours dans un milieu francophone ${ }^{\dagger}$

\begin{tabular}{|c|c|c|c|c|c|}
\hline \multirow[b]{2}{*}{ Durée des séjours } & \multicolumn{2}{|c|}{ Je vais } & \multicolumn{2}{|c|}{ Je vas } & \multirow[b]{2}{*}{ TOTAL } \\
\hline & $\mathrm{N}$ & $\%$ & $\mathrm{~N}$ & $\%$ & \\
\hline O jour-I jour & I 2 & 75 & 4 & 20 & I6 \\
\hline $2-7$ jours & 7 & 77 & 2 & 22 & 9 \\
\hline $8-2$ I jours & 33 & 89 & 2 & 5 & 37 \\
\hline$>3$ semaines & 29 & IOO & $\mathrm{O}$ & $\mathrm{o}$ & 29 \\
\hline Total & $8 \mathrm{I}$ & 85 & 8 & I 5 & 95 \\
\hline
\end{tabular}

${ }^{\dagger}$ Ces données viennent de Mougeon et Nadasdi (1998) et se basent sur un souséchantillon de 24 locuteurs.

En bref, notre étude de la variation je vais/vas/m'as apporte une confirmation supplémentaire à la tendance générale formulée au début de la section 3: les élèves d'immersion font un usage marginal ou nul des variantes vernaculaires, sur-utilisent les variantes formelles et emploient des formes qui sont identiques à certains des usages non standard des locuteurs Li mais qui, dans leur parler, ont de toute évidence une origine développementale.

\section{IO CONCLUSION}

Dans presque toutes les études sur l'acquisition de la variation sociostylistique du français par les apprenants avancés du français L2 en contexte éducationnel, on a trouvé que ces apprenants avaient un répertoire de variantes plus restreint que les locuteurs Li et qu'ils employaient les variantes à des niveaux de fréquence différents de ceux qu'on pouvait observer dans le discours des locuteurs Li. On a invoqué plusieurs facteurs pour expliquer cette quasi-constante: i) le contexte scolaire n'offre pas une gamme de situations de communication assez large et diversifiée; ii) les enseignants et les manuels font un traitement sélectif de la variation (ils favorisent ou excluent certaines variantes); et iii) pour des raisons intersystémiques et/ou intrasystémiques certaines variantes sont particulièrement difficiles à acquérir.

Ce n'est pas ce que nous avons trouvé dans notre étude générale de l'acquisition des variantes du futur par les élèves d'immersion. En effet, d'une part ces élèves emploient les trois variantes que l'on retrouve dans le français parlé des locuteurs Li du français québécois et d'autre part, la fréquence d'emploi de ces variantes dans le parler de nos élèves est relativement semblable à celle observée dans le parler des locuteurs Lı. Ce résultat est probablement attribuable à quatre facteurs: i) les enseignants d'immersion emploient en salle de classe les trois variantes du futur sensiblement au même niveau de fréquence que les locuteurs Li en situation d'entrevue enregistrée; ii) la variante favorisée par les francophones (le 
périphrastique) est analytique plutôt que synthétique; iii) le futur périphrastique et le futur présent ressemblent aux formes qui expriment le futur en anglais; et iv) le futur fléchi fait l'objet d'un emploi privilégié dans les manuels de français. Le fait que les enseignants rejoignent les locuteurs LI relativement à la fréquence des variantes est lui aussi inhabituel. C'est la première fois dans nos recherches que nous avons observé une pareille convergence. Elle est probablement pour partie attribuable au fait que dans le cas de variation qui nous intéresse ici la fréquence des variantes ne fluctue pas en fonction de l'appartenance socio-économique des locuteurs en français canadien parlé. Reste à voir si dans nos recherches futures nous trouverons un autre cas de variation où la compétence variationnelle des élèves d'immersion est similaire à celle des locuteurs Li et si l'on peut à nouveau invoquer certaines des explications mentionnées plus haut.

Notre recherche a montré que les élèves d'immersion ne respectent qu'une seule des six contraintes linguistiques observées par les locuteurs du français québécois, à savoir la contrainte reliée à la présence d'un adverbe de temps. Parmi les contraintes qui ne sont pas respectées par les élèves d'immersion, on doit mentionner celle qui est reliée aux phrases négatives (contexte syntaxique favorable à l'emploi du futur fléchi), car elle est la contrainte linguistique 'no I' en français québécois Li. Notre étude des contraintes linguistiques associées à la variable futur apporte donc une confirmation supplémentaire du fait que les élèves d'immersion maitrisent mal les contraintes linguistiques de la variation. À ce sujet, des recherches portant sur des groupes d'apprenants plus avancés que les élèves d'immersion sur lesquels porte notre recherche, font état d'une meilleure maitrise des contraintes linguistiques de la variation (cf. p. ex. Blondeau et Nagy, I998; Sax, I999). Ceci dit, étant donné que les cas de variation étudiés avec des corpus d'apprenants avancés et des corpus d'apprenants moins avancés sont rarement les mêmes, il est peut-être trop tôt pour attribuer cette différence au fait que les apprenants plus avancés ont été plus exposés à la langue cible (notamment par le biais de contacts avec les locuteurs Li).

Notre recherche a montré que trois paramètres sociaux influent sur l'emploi des variantes du futur par nos élèves: i) le sexe (les filles emploient le futur fléchi plus souvent que les garçons); ii) la durée des séjours en milieu francophone; et iii) le niveau de scolarité (l'effet de ces deux facteurs se traduit par une sur-utilisation de la variante périphrastique par les élèves de I $2^{\text {ème }}$ année et par ceux qui ont effectué les plus longs séjours).

En ce qui concerne l'association entre le sexe féminin et l'emploi du futur fléchi (variante associée au registre formel ${ }^{11}$ ) on peut remarquer qu'il confirme la tendance dégagée par Rehner, Mougeon et Nadasdi (à paraitre). Ceci dit, on doit préciser que l'association entre le sexe féminin et l'emploi préférentiel des variantes formelles n'est pas une constante autant dans le domaine des études sur l'acquisition de la variation du français par les élèves d'immersion (cf. section 3) que dans la recherche sur l'acquisition de cette variation par d'autres apprenants du français L2

${ }^{11}$ Il se peut aussi que les manuels jouent un rôle important dans le style d'apprentissage des filles. 
(cf. p. ex. Dewaele et Regan (200I; 2002), qui n'ont trouvé d'effet du paramètre sexe ni sur l'effacement de ne par des apprenants néerlandophones, ni dans leur emploi de lexèmes familiers). Pour ce qui est de l'effet positif des facteurs séjour en milieu francophone et niveau de scolarité sur l'emploi du futur périphrastique, on peut remarquer qu'il confirme une tendance déjà bien établie (cf. section 3): les contacts avec les locuteurs $\mathrm{L}_{\mathrm{I}}$ en dehors de l'école favorisent l'acquisition des variantes qui font partie de l'usage courant de ces locuteurs. Dans la présente étude on a pu constater que ces contacts avaient un effet positif sur une variante courante qui n'est pas contraire à l'usage standard alors que dans les recherches antérieures cet effet a été observé relativement à des variantes courantes et non standard.

Notre micro-étude consacrée aux variantes du futur périphrastique de la I ère personne du singulier a révélé que les élèves n'utilisent pas du tout la variante vernaculaire $m$ 'as + infinitif, emploient peu souvent je vas + infinitif et favorisent massivement la variante formelle je vais + infinitif. Ce résultat est conforme à une tendance observable dans plusieurs recherches sur l'acquisition de la variation sociostylistique par les apprenants avancés du français L2 en contexte scolaire (y compris les élèves d'immersion, cf. section 3): ces apprenants se distinguent des locuteurs Li par un emploi marginal des variantes vernaculaires et une surutilisation des variantes standard ou formelles.

Notre micro-étude a aussi trouvé que les contacts avec les locuteurs Li n'ont pas d'effet positif sur l'acquisition des variantes $m$ 'as et je vas. En effet, on a vu que les élèves qui ont séjourné le plus longtemps en milieu francophone n'emploient aucune de ces deux variantes. D'après nous, ce résultat reflète probablement pour partie le fait que les séjours de ces élèves n'ont duré que 59 jours en moyenne, et donc, somme toute, que leur longueur est relativement modeste. Ceci dit, avec d'autres cas de variantes informelles (p. ex. l'effacement de $n e$, l'emploi du pronom sujet on à la I ère personne du pluriel), nous avons trouvé que ces mêmes élèves employaient plus souvent ces deux variantes que les autres élèves. En d'autres termes, il semblerait que l'effet positif des contacts avec les locuteurs LI du français dépende des propriétés des variantes (fréquence discursive, saillance de leur marque sociale ou stylistique, etc.) auxquelles sont exposées les apprenants par le biais des contacts avec les locuteurs LI. Regan (I996) est arrivée à une conclusion similaire lorsqu'elle a constaté qu'après un an de séjour en France, dans le parler de leurs apprenants l'effacement de la particule ne (variante informelle très récurrente) était presque aussi fréquent que dans le parler des locuteurs Li du français, alors que ses apprenants ne faisaient qu'un usage modeste des lexèmes typiques du registre familier (variantes plus marquées).

Finalement, à la fois notre micro-étude des variantes du futur périphrastique et notre étude générale de la variante «futur» ont montré que la prise en compte de l'input éducationnel des apprenants (la langue des enseignants et des manuels) jetait un éclairage intéressant sur la compétence variationnelle des élèves d'immersion. Il est à souhaiter que d'autres chercheurs examineront le rôle de ces facteurs dans l'acquisition de la variation par les apprenants des langues secondes, car dans 
notre propre recherche ces facteurs se sont avéré jouer un rôle important (cf. section 3).

Authors' addresses:

T. Nadasdi

Modern Languages and Cultural Studies

University of Alberta

Edmonton, Alberta

Canada

K. Rehner

Modern Languages Centre

OISE/University of Toronto

Toronto, Ontario

Canada

\author{
R. Mougeon \\ French Studies \\ York University \\ Toronto, Ontario \\ Canada
}

\section{RÉFÉRENCES}

Allen, P., Cummins J., Harley, B. et Swain, M. (1987). Development of Bilingual Proficiency Project. Toronto: OISE/UT.

Andersen, R. (I990). Models, processes, principles and strategies: second language acquisition inside and outside the classroom. In: B. Van Patten and J. Lee (eds.), pp. $45-68$.

Bartning, I. (1998). Procédés de grammaticalisation dans l'acquisition des prédications verbales en français parlé. Travaux de linguistique, 36: 223-234.

Bayley, R. et Preston, D. (Dir.) (I996). Second Language Acquisition and Linguistic Variation: Studies in Bilingualism, 10. Philadelphie: Benjamins.

Blondeau, H. et Nagy, N. (I998). Double marquage du sujet dans le français parlé par de jeunes Anglo-Montréalais. Les Actes de la conférence de l'ACL. Cahiers linguistiques d'Ottawa: 59-70.

Deshaies, D. et Laforge, E. (I98I). Le future simple et le futur périphrastique dans le français parlé dans la ville de Québec. Langues et linguistique, 7: 23-37.

Dewaele, J.-M. et Regan, V. (200I). The use of colloquial words in advanced French interlanguage. In: S. Foster-Cohen and A. Nizegorodcew (eds.), EUROSLA Yearbook (200I). Amsterdam - Philadelphia: Benjamins, pp. 5I-68.

Dewaele, J.-M. et Regan, V. (2002). Maîtriser la norme sociolinguistique en interlangue française: le cas de l'omission variable de ne. Journal of French Language Studies, I2.I: I $23-$ I 68 .

Emirkanian, L. et Sankoff, D. (1985). Le futur simple et le futur périphrastique dans le français parlé. In: M. Lemieux et H. Cedergren (Dir.), Les Tendances dynamiques du français parlé à Montréal 2. Québec: Gouvernement du Québec, pp. I 89-204.

Harley, B. (I992). Patterns of second language development in French immersion. Journal of French Language Studies, 2.2: I 59-183.

Harley, B. et King, M. L. (1989).Verb lexis in the written compositions of young L2 learners. Studies in Second Language Acquisition, I I.4: 4I 5-436.

King, R. et Nadasdi, T. (à paraître). Back to the Future in Acadian French. Journal of French Language Studies. 
Knaus, V. et Nadasdi, T. (200I). Etre ou ne pas être in Immersion French. The Canadian Modern Language Review, 58.2: 286-306

Labov, W. (1990). The intersection of sex and social class in the course of linguistic change. Language Variation and Change, I I.2: $205-254$.

Lapkin, S., Hart, D. and Swain, M. (1995). A Canadian interprovincial exchange: Evaluating the linguistic impact of a three-month stay in Quebec. In: B. F. Freed (ed.), Second Language Acquisition in a Study Abroad Context. Philadelphia: Benjamins, pp. 67-94.

Lesage, R. et Gagnon, S. (1992). Futur simple et futur périphrastique dans la presse Québécoise. In: A. Crochetière, J.-C. Boulanger et C. Ouellon (éds.), Actes du XV $V^{\text {ème }}$ Congrès International des Linguistes. Québec: Les Presses de l'Université Laval, pp. 367370.

Lyster, R. (1994). The effect of functional-analytic teaching on aspects of French immersion students' sociolinguistic competence. Applied Linguistics, I 5: 263-287.

Meisel, J. (1983). Strategies of second language acquisition: more than one kind of simplification. In: R. Andersen (ed.), Pidginization and Creolization as Language Acquisition. Rowley, MASS.: Newbury house, pp. I20-I 57.

Mougeon, R. (1996). Recherche sur les origines de la variation vas, m'as, vais en français québécois. In: T. Lavoie (éd.), Français du Canada - Français de France. Tübingen: Niemeyer, pp. 60-77.

Mougeon, R. et Beniak, É. (I99I). Linguistic Consequences of Language Contact and Restriction: The Case of French in Ontario. Oxford: Oxford University Press.

Mougeon, R. et Nadasdi, T. (1998). Use of Analytic and Synthetic Verb Forms to Express the Future Time in the Spoken French of High School French Immersion Students. Communication à la conference: Trends in Second Language Teaching and Learning, Ottawa (Ontario).

Mougeon, R., Nadasdi, T. et Rehner, K. (à paraître). Acquisition de la variation par les apprenants avancés du français: les élèves d'immersion. Dans un numéro spécial de la revue AILE, édité par J.-M. Dewaele et R. Mougeon.

Mougeon, R. et Rehner, K. (200I). Variation in the spoken French of Ontario French immersion students: The case of juste vs seulement vs rien que. Modern Language Journal, 85.3: 398-4I5.

Nadasdi, T. (200 I). Agreeing to disagree: Variable subject verb agreement in immersion French. The Canadian Journal of Applied Linguistics, 4. I-2: 87-IOI.

Nadasdi, T. et McKinnie, M. (sous presse) Living and working in immersion French. Journal of French Language Studies.

Poplack, S. et Turpin, D. (I999). Does the FUTUR Have a Future in (Canadian) French? Probus Numéro spécial: I34-I64.

Poplack, S. (1989). The Care and Handling of a Mega-Corpus: the Ottawa-Hull French Project. In: R. Fasold and D. Schiffrin (eds.), Language Change and Variation. Amsterdam/Philadelphia: John Benjamins.

Rand, D. et Sankoff, D. (I990). GoldVarb version 2: A variable rule application for the Macintosh. Centre de recherches mathématiques, Université de Montréal.

Regan, V. (I996). Variation in French interlanguage: a longitudinal study of sociolinguistic competence. In: R. Bayley et Preston, D. (eds.), Second Language Acquisition and Linguistic Variation. Amsterdam-Philadelphia: John Benjamins, pp. I77201 . 
Rehner, K. et Mougeon, R. (1999). Variation in the spoken French of immersion students: To ne or not to ne, that is the sociolinguistic question. La Revue canadienne des langues vivantes, 56.I: I24-I54.

Rehner, K. and Mougeon, R. et Nadasdi, T. (200I). Expressing the notion of consequence in immersion French. NWAV 30, Raleigh, Oct. I I-I4, 200 I.

Rehner, K. and Mougeon, R. et Nadasdi, T. (à paraître). The learning of sociostylistic variation by advanced FSL learners: the case of nous versus on in immersion French. Studies in Second Language Acquisition.

Sabeau-Jouannet, E. (I977). L'expression de l'organisation spatiale et temporelle, son évolution chez l'enfant de 2 à 5 ans. In: F. François, D. François, E. Sabeau-Jouannet et Sourdot, M. (eds.), Syntaxe de l'enfant avant 5 ans. Paris: Larousse, pp. 193-203.

Sankoff, G. et Thibault, P. (1980). The alternation between the auxiliaries avoir and être in Montréal French. In: G. Sankoff (ed.), The Social Life of Language. Philadelphia: University of Pennsylvania Press, pp. 2 I I-345.

Sax, K. (1999). Acquisition of stylistic variation by American learners of French: the case of ne deletion. Communication à la $28^{\text {ème }}$ conférence NWAV, Toronto, (Ontario), I 4 -I 8 octobre.

Swain, M. and Lapkin, S. (I990). Aspects of the sociolinguistic performance of early and later French Immersion students. In: R.C. Scarcella, E.S. Anderson and S.D. Krashen (eds.), Developing Communicative Competence in a Second Language. New York: Newbury House, pp. 4I-54.

Tarone, E. et Swain, M. (I995). A sociolinguistic perspective on second language use in immersion classrooms. Modern Language Journal, 79.2: I66-I 78.

Uritescu, D., Nadasdi T., Mougeon, R. et Rehner, K. (200I). A sociolinguistic analysis of phonetic variation in the spoken French of immersion students. Communication à la réunion annuelle de l'Association canadienne de linguistique appliquée, Université Laval, Québec, mai.

Van Patten, B. (1990). The acquisition of clitic pronouns in Spanish: two case studies. In: B. Van Patten and J. Lee (eds.), pp. I I 8-i39. 